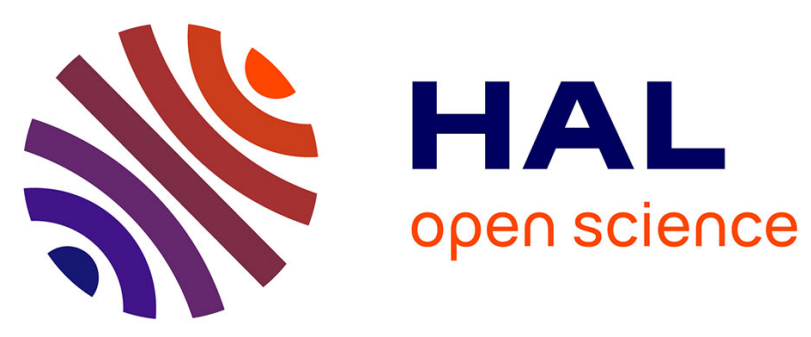

\title{
Vivre dans les programmes
}

\author{
Anthony Masure
}

\section{To cite this version:}

Anthony Masure. Vivre dans les programmes. Multitudes, 2019, Pour l'Europe, 74, pp. 176-181. hal-02924481

\section{HAL Id: hal-02924481 \\ https://hal.science/hal-02924481}

Submitted on 28 Aug 2020

HAL is a multi-disciplinary open access archive for the deposit and dissemination of scientific research documents, whether they are published or not. The documents may come from teaching and research institutions in France or abroad, or from public or private research centers.
L'archive ouverte pluridisciplinaire HAL, est destinée au dépôt et à la diffusion de documents scientifiques de niveau recherche, publiés ou non, émanant des établissements d'enseignement et de recherche français ou étrangers, des laboratoires publics ou privés. 


\title{
VIVRE DANS LES PROGRAMMES
}

\author{
Anthony Masure
}

Association Multitudes | « Multitudes »

2019/1 n 74 | pages 176 à 181

ISSN 0292-0107

Article disponible en ligne à l'adresse :

https://www.cairn.info/revue-multitudes-2019-1-page-176.htm

Distribution électronique Cairn.info pour Association Multitudes.

(C) Association Multitudes. Tous droits réservés pour tous pays.

(cc) BY-NC 


\section{Vivre dans les programmes Anthony Masure}

Tour à tour linguiste, philosophe, théoricien des médias, anthropologue, artiste, biologiste, Vilém Flusser (1920-1991) aura autant traversé les continents que les frontières de la pensée, devenant ainsi un intellectuel migrant ( «bodenlos», littéralement «sans sol ferme» en allemand) comme purent l'être en leur temps Walter Benjamin ou Hannah Arendt. Naviguer dans ces méandres nécessite d'explorer des textes originellement rédigés en portugais, allemand, anglais et français, traduits par Flusser dans une démarche d'auto-distanciation (il repensait les plans et contenus des ouvrages dans leur langue d'accueil) ou de façon posthume.

Ces strates linguistiques ne se complètent que partiellement, ce qui explique la difficulté à saisir la complexité et la richesse de sa pensée. Les lecteurs portugais sont familiers du Flusser linguiste ou exégète des religions, et les allemands, du théoricien des médias. Les anglophones, sous l'impulsion de théoriciens des médias et d'artistes comme Siegfried Zie- linski, Andreas Ströhl ou Andreas MüllerPohle, connaissent davantage les lumineux essais (traduits de l'allemand) Into the Universe of Technical Images ([1985] 2011) et Does Writing Have a Future? ([1987] 2011), ainsi que les essais comme Natural: Mind ([1979] 2013) ou Post-History ([1983] 2013) traduits du portugais par Rodrigo Maltes Novaes, artiste, traducteur, éditeur et assistant de Flusser ${ }^{1}$. Mentionnons également les récentes rééditions de textes anglais méconnus, tels que les Artforum essays aux éditions Metaflux (2017).

Les lecteurs francophones n'ont, à l'heure actuelle, accès qu'à des recueils traduits depuis l'anglais ou l'allemand ${ }^{2}$ : La force du quotidien (Mame, 1973, remanié depuis l'allemand sous le titre Choses et non choses), Pour une philosophie la photographie (Circé, 1998), Les Gestes

1 Tous ces textes sont édités par University of Minnesota Press / Univocal Publishing.

2 Par soucis d'économie, nous n'indiquons entre parenthèses que les dates des premières publications et/ou traductions en langue française. 


\section{Mineure Vilém Flusser : vivre dans les programmes}

(Hors Commerce, 1999), Petite philosophie du design (Circé, 2002), Essais sur la nature et la culture (Circé, 2005), La civilisation des médias (Circé, 2006), ou encore l'étrange Vampyroteuthis Infernalis ${ }^{3}$ (Zones Sensibles, 2015). En plus d'articles publiés dans des revues comme Communication \& Langages et Théâtre/Public, Cause commune, etc., les rares textes de Flusser écrits en français et rendus publics (à l'époque) sont, à notre connaissance, la conférence Le monde codifié (Institut de l'Environnement, 1974) et des communications rédigées pour le «paranaturaliste» Louis Bec dans les années 1970 (notices d'expositions, etc.).

\section{Flusser, écrits français}

De même qu'il existe un Benjamin "français ${ }^{4}$ », le Flusser français reste donc majoritairement méconnu. Publié en 2015, le glossaire critique Flusseriana: An Intellectual Toolbox ${ }^{5}$ comprend 200 entrées rédigées en anglais, allemand et portugais. Chacune des trois langues occupe une colonne du livre. Une quatrième colonne, vide, rend ainsi involontairement compte du manque de réception, en France, de l'amplitude des travaux de Flusser ${ }^{6}$. De façon similaire, le site web FlusserBrasil ${ }^{7}$ comporte un item vide de «textes en français» - seuls les textes allemands, anglais et portugais sont en ligne.

3 Yves Citton, «Naviguer ou filtrer. Vilém Flusser et l'alternative vampirique de l'imaginaire numérique», Hybrid, $\mathrm{n}^{\circ} 3$, 2016, www.hybrid.univ-paris8.fr/lodel/index. php?id=615

4 Écrits français [1933-1940], Folio, 2003.

5 Siegfried Zielinski, Peter Weibel, Daniel Irrgang (dir.), Flusseriana: An Intellectual Toolbox, University of Minnesota Press, Univocal, 2015.

6 Pour un aperçu général de l'œuvre de Flusser, voir: Anke Finger, Rainer Guldin, Gustavo Bernardo, Vilém Flusser: An Introduction, University of Minnesota Press, coll. «Electronic Mediations», 2011.

7 www.flusserbrasil.com
Enfin, la foisonnante revue Flusser Studies, sur une liste de 210 auteurs, mentionne comme seuls contributeurs francophones Louis Bec, Annick Bureaud, Fred Forest, Marc Lenot, et Jamine Marchessault (Canada).

La consultation de ses archives prend alors tout son sens et éclaire les multiples facettes de son œuvre'. Rassemblés à la mort de Flusser en 1991 par son épouse Edith Flusser, les archives «nomades» de Flusser ont tout d'abord été conservées à La Haye à partir de 1992. Elles ont déménagé avec elle à Munich, puis ont été confiées au chercheur Siegfried Zielinski qui les a installées à l'Académie des arts médiatiques de Cologne de 1998 à 2006. Depuis 2007, les originaux sont conservés à Cologne tandis qu'une copie sous forme de fac-similés est consultable publiquement à l'université des Arts de Berlin $(\mathrm{UdK})^{10}$. Dirigée par Maren Hartmann et gérée par Anita Jóri, l'archive Flusser de Berlin contient également la bibliothèque personnelle de Flusser, des documents audiovisuels, de même que de nombreux travaux réalisés autour de son ouvre. Au-delà de permettre une consultation facile de ces documents, l'archive a été pensée comme un lieu de travail, un $h u b$ de personnes aux nationalités et parcours multiples ${ }^{11}-$ une archive vivante et autocritique, à l'image de Flusser.

\footnotetext{
8 www.flusserstudies.net

9 Ces recherches personnelles ont pu être entreprises grâce au dispositif «Soutien à la recherche en théorie et critique d'art» du Centre national d'arts plastiques - CNAP (session 2017).

10 Une autre instance des fac-similés est consultable depuis 2012 à São Paulo (Brésil), au Center for Interdisciplinary Research in Semiotics of Culture and Media (CISC, rattaché à l'Université Pontificale Catholique de São Paulo) fondé en 1992 sous la direction de Norval Baitello Jr.

11 Voir à ce propos la foisonnante revue Flusser Studies: www.flusserstudies.net
} 
À notre connaissance peu fréquentée par des chercheurs francophones ${ }^{12}$, l'archive Flusser n'est guère mentionnée, en français, que dans la préface rédigée par la chercheure Sandra Parvu pour la précise réédition augmentée des Gestes dirigée par Marc Partouche ${ }^{13}$, désormais épuisée. Sur un plan intellectuel, il est ainsi étrange d'avoir traduit depuis l'anglais ou l'allemand des textes parfois disponibles en français de la main de l'auteur - ce qui rappelle les querelles de la French Theory ou des Visual Studies, aux écrits redécouverts après un détour depuis d'autres pays. Cette situation est d'autant plus problématique que les concepts reformulés par Flusser dans chaque langue sont ainsi perdus, sans même parler de l'absence d'appareils critiques nuisibles à la compréhension des contextes de rédaction.

Rédigés à partir du milieu des années 1970 (en mai 1975, il s'installe à La Tour d'Aigues en Provence et en 1980, il achète une maison dans le village de Robion ${ }^{14}$ ), les écrits français de Flusser (une soixantaine d'articles sont conservés dans l'archive) sont à la fois des traductions personnelles d'autres travaux, mais également des contenus originaux, notamment des cours de théorie de la communication donnés à l'école d'art de Marseille-Luminy. L'étude de sa correspondance française montre de plus les nombreux liens qu'il entretenait avec des revues, éditeurs (Denoël, Gallimard) et chercheurs (Abraham Moles, par exemple). À une époque où les mails et la bureaucratie de la recherche n'existaient pas encore, ces

12 Constat partagé par le chercheur Riccardo Venturi dans: "Les Images techniques et leur médium. Exposer la pensée de Vilém Flusser », Critique d'art, n 46, printemps/été 2016, http://journals.openedition.org/critiquedart/21166

13 Al Dante/Aka, 2014.

14 Merci à Marc Lenot pour ces précisions et pour la relecture de ce texte. demandes de publications, lettres de refus, factures en retard, montrent l'urgence qu'avait Flusser à partager ses pensées: il disait ainsi que «nous survivrons par la mémoire des autres».

\section{Fulgurantes provocations}

Sans doute trop en avance sur leur temps, les idées provocatrices de Flusser lui valaient souvent de l'incompréhension voire de l'hostilité. Relire ou découvrir aujourd'hui les écrits de Flusser, parfois à près de quarante ans d'écart, c'est faire l'expérience d'un saisissement. Il est tout d'abord stupéfiant de constater la capacité d'anticipation de Flusser, qui entrevoyait des développements techniques insoupçonnés dans les années 1970 (son idée d'une "communication dialogique» électronique sera par exemple partiellement réalisée dans le développement du Web à partir de 1989), et dont certains restent encore obscurs pour les nonspécialistes (biotechnologies avec sa notion d' «art vivant», systèmes auto-apprenants à la façon du deep learning, "instruments intelligents», etc.). Esprit libre, Flusser ne s'embarrassait pas de citations et de notes de bas de page par soucis d'efficacité. Conservée à Berlin, sa «bibliothèque de voyage» montre pourtant la pertinence avec laquelle il se documentait: le voisinage d'exemplaires de la revue de contre-culture Radical Software (1970-1974) avec des manuels techniques ou des livres de Günther Anders, Hannah Arendt, Ernst Cassirer, témoigne d'une mobilité d'esprit qui ferait pâlir les revendications contemporaines du «décloisonnement» des disciplines.

Il est également frappant de constater que les écrits ne sont qu'une face du travail de Flusser, qui aura multiplié les collaborations avec des artistes comme Fred Forest ou Harun 


\section{Mineure Vilém Flusser : vivre dans les programmes}

Farocki ${ }^{15}$. En contact avec le designer Karl Gerstner (auteur de l'essai Designing programmes, 1964), il imagine en 1988 la Casa da Côr, une maison cybernétique d'encodage chromatique des connaissances humaines. En 1987, Flusser réalise une version électronique (sur disquette) de son essai Does writing have a future? aux éditions Immatrix. L'intention était d'établir un dialogue avec les lecteurs, qui pouvaient renvoyer à l'auteur leurs propres versions ${ }^{16}$. Toujours à la fin des années 1980, Flusser modifiait le logiciel HyperCard d'Apple (1987) pour créer une collection de fiches aux multiples modes de relations. Ce «Flusser Hypertext» a depuis été émulé et est désormais disponible sur le Web.

Ces projets montrent l'importance, dans l'œuvre de Flusser, de la pratique des environnements électroniques. On retrouve cette conscience matérialiste chez des théoriciens des médias comme Friedrich Kittler (19432011), pour qui la connaissance concrète du fonctionnement interne des machines électroniques est un préalable obligatoire à l'analyse de leurs enjeux. Plus proches de nous, des chercheurs comme Lev Manovich (artiste et informaticien) ou Wolfgang Ernst («archéologue des médias») demandent à leurs étudiants de maîtriser a minima les logiques de programmation. C'est cette notion de "programme», essentielle dans la pensée de Flusser, que se propose d'explorer ce dossier de Multitudes de textes écrits en français, pour la plupart inédits, sélectionnés et annotés par nos soins.

15 Voir notamment l'exposition «Without Firm Ground - Vilém Flusser and the arts» et son catalogue associé, ZKM Karlsruhe, 2015, https://zkm.de/en/exhibition/2015/08/globale-without-firm-ground-vilem-flusser-and-the-arts

16 Rainer Guldin, «Derrida and Flusser: On the Concept of Writing and the End of Linearity», Annual MLA Convention Philadelphia (USA), 2004.

\section{Les programmes de Flusser}

Le concept de programme nous intéresse particulièrement en raison de ses conséquences anthropologiques, que nous avons encore bien du mal à déplier. De façon générale, nous pouvons définir la notion de programme comme ce qui est écrit à l'avance (programme), c'est-à-dire comme un ensemble de règles logiques visant à effectuer une tâche. Les programmes numériques sont la traduction en langages formels (de programmation) des notations génériques des algorithmes. Le terme «algorithme» tire son origine du nom du mathématicien arabe al-Kwharizmi (783850) traduit phonétiquement en Algaurizin, et du mot grec arithmos, nombre. Flusser fait ainsi entendre dans ses écrits la dimension séculaire, voire millénaire, du programme qu'il rapproche de la notion de calcul via les calculi, ces petits cailloux dont les logiques se retrouvent dans nos machines siliconées.

Ayant très vite pris conscience des bouleversements considérables introduits par la discrétisation de l'écriture linéaire en unités calculables, Flusser développe une pensée de la technique visant à nous alerter sur la puissance des "appareils» qu'il comprend, à la façon des «dispositifs" d'Agamben, comme des puissances d'automatisation et d'aliénation des subjectivités humaines. Ces «boîtes noires» (Filosofia da Caixa Prêta, du titre en portugais du Brésil de l'essai Pour une philosophie la photographie) sont d'autant plus problématiques qu'elles sont également opaques pour les "programmeurs", ceux qui écrivent le code, les programmeurs étant eux-mêmes programmés par des appareils de niveau supérieur. Flusser émet ainsi l'idée d'appareils engendrant des appareils sans intervention humaine, rejoignant ainsi les interrogations 
contemporaines autour des programmes mutants et des intelligences artificielles comparables à des organismes vivants.

Trouvant son apogée dans Auschwitz, le "programme occidental», selon Flusser, fait de nous des «fonctionnaires», c'est-à-dire des agents ne faisant que manipuler des symboles et traitant toute chose ou personne comme une entité objectivable et calculable, rejoignant ainsi la pensée d'auteurs comme Günther Anders ou Hannah Arendt. La logique formelle des programmes se substitue à la fois à la pensée finaliste (magique, religieuse) et à la pensée causale (scientifique), en imposant un cadre de pensée dans lequel les notions de liberté et de politique ne sont plus opérantes. Flusser a bien conscience de l'impossibilité de s'attaquer directement aux programmes car ceux-ci sont indissociables de notre culture; toute critique est donc vouée l'échec. Si leur destruction est impossible, il nous faut donc apprendre à vivre dans les programmes.

\section{Virtualités dialogiques}

Rétrospectivement, on ne peut qu'être frappé de la force des intuitions de Flusser, qui rejoignent celles des rares penseurs, tels que Félix Guattari, à avoir perçu dès les années 1980 les profondes implications des machines électroniques. Mais les analyses de Flusser ne sont pas derrière nous, loin s'en faut, tandis qu'en ce premier quart $\mathrm{du} \mathrm{XXI}^{\mathrm{e}}$ siècle la transformation des subjectivités en sujets par des "prescriptions» (programmes) ne semble jamais avoir été aussi vive. Nous sommes au cœur du paradoxe énoncé par Flusser: nous préférons être programmés, car que ferons-nous si nous ne le sommes plus? Dans les intervalles que cherchent à combler les modélisations calculées, nous guettent l'ennui et l'oubli de notre finitude.
Cette liberté au sein des mondes artificiels, qui reste à construire, est à relier au concept de virtualité. Pour Flusser, la programmation n'est que la réalisation des différentes potentialités («virtualités») contenues dans les programmes, à la manière du génome biologique. Ainsi, pour Flusser, l'artiste-photographe est celui qui parvient à réaliser de nouvelles virtualités (informations) dans ce que permet l'appareil-photo, et qui par-là, en quelque sorte, le dé-programme. La «vie artificielle» qu'appelle Flusser n'est donc pas dépourvue de programmes. Elle n'est pas non plus une société où chacun serait programmeur, car la complexité technique d'une telle visée semble insurmontable. Selon Flusser, c'est dans les notions de jeu et de hasard, soit donc, dans l'acceptation du caractère absurde et non discrétisable de l'existence humaine, qu'une issue est possible. $\mathrm{Si}$ nous devons vivre dans les programmes, c'est bien de programmes «dialogiques» dont nous avons besoin. Cette problématique notion de dialogue - qui tend à disparaître du Web à mesure que ce dernier, sous le poids de l'appétit des GAFAM, se verticalise en TV (en apps) nous semble, en creux, incarner la méthode d'écriture de Flusser. La brièveté de ses textes et leurs multiples contradictions et provocations peuvent ainsi se comprendre comme une façon d'offrir au lecteur un espace où intercaler ses réflexions. Dans la préface de la version française (inédite) de son essai Post-Histoire, Flusser explique que ses écrits doivent être compris comme des «modes d'emploi », expression que l'on pourrait rapprocher de la dimension prescriptive des programmes. Mais, ajoute-t-il juste après, «le titre "mode d'emploi" est une farce bien sûr. Il n'est question ni de prescriptions ni d'être consommé. Le mode d'emploi, une fois lu, doit être jeté, et que le lecteur fasse l'usage du texte qu'il veut». 


\section{Mineure Vilém Flusser : vivre dans les programmes}

Face au fonctionnement pyramidal des mass media et au torrent abrutissant des «techno-images», les artistes peuvent au contraire œuvrer au développement d'une nouvelle imagination, d'un "techno-imaginaire» capable de donner du sens à cette vie artificielle, à cette vie dans l'ennui des intervalles. Observer comment travaillait Flusser est à ce titre instructif. Il faut souligner la dimension phénoménologique de ses écrits, à savoir sa capacité à regarder de façon singulière le monde qui l'entoure: stylos, télévision, vaches, pluie, gestes, etc. Comparables aux écrits du poète Francis Ponge ${ }^{17}$ ou des designers Bruno Munari ${ }^{18}$ et Alessandro Mendini ${ }^{19}$, les observations de Flusser engagent un dialogue intérieur avec le lecteur en bousculant la stabilité de ce qu'il pensait tenir pour acquis. Il nous reste à inventer une phénoménologie des programmes. 